\title{
Fungi as a promising tool for bioremediation of soils contaminated with aromatic amines, a major class of pollutants
}

Philippe Silar, Julien Dairou, Angélique Cocaign, Florent Busi, Fernando Rodrigues-Lima and Jean-Marie Dupret

In their recent article, (Untapped potential: exploiting fungi in bioremediation of hazardous chemicals. Nature Rev. Microbiol. 9, 177-192 (2011)) $)^{1}$, Harms et al. highlight that the potential use for fungi in bioremediation has not received the attention it deserves. We fully agree with Harms et al. and wish to further highlight the promising metabolic capabilities of these organisms in the remediation of a major class of pollutants, the aromatic amines (AA).

In a pilot study, we provided proof-ofconcept remediation experiments in which Podospora anserina, through its arylamine $\mathrm{N}$-acetyltransferase 2 (NAT2) enzyme, detoxifies the highly toxic pesticide residue 3,4-dichloroaniline (3,4-DCA) in experimentally contaminated soil samples ${ }^{2}$. 3,4-DCA is the major breakdown product of the phenylurea herbicides diuron and linuron and of the anilide propanil. It belongs to the class of AA, an important and diversified class of soil pollutants. Many AA are toxic to most living organisms ${ }^{3}$. In particular, AA account for $12 \%$ of the 415 chemicals known or strongly suspected to be carcinogenic in humans ${ }^{4}$. Some aniline derivatives, such as 3,4-DCA or 3,5-DCA, are persistent in soils and waters and exhibit potential toxicity ${ }^{5,6}$. Residues of sulphonamides, an important class of AA drugs, have been detected in manure at levels of up to $12 \mathrm{mg}$ per $\mathrm{kg}^{7}$. The ecotoxicological effects of these drug contaminations remain to be studied more thoroughly. However, toxic effects have already been reported ${ }^{8,9}$. AA contamination can involve industrial chemicals, some of which, including azo dyes, have been shown to be toxic ${ }^{10}$. Soils can also be contaminated by toxic nitroaromatic compounds ${ }^{11}$.

Exploiting the ability of microorganisms to transform AA pollutants is a promising approach for bioremediation. Most studies have focused on conversion of 3,4-DCA into its acetylated form. First, it has been shown that acetylated 3,4-DCA is less toxic than 3,4-DCA; second, some soil bacteria and fungal strains acetylate 3,4-DCA ${ }^{12,13}$. Although aniline derivatives undergo complex transformations in soils, these studies open up new possibilities in bioremediation.

The major detoxification pathway of AA depends on the activity of NATs ${ }^{14}$. NATs affect the bioavailability of many AA drugs and carcinogens. Using the potential degradative properties of NATs expressed in soil microorganisms, we explored putative AA bioremediation pathways using soil bacteria or filamentous fungi ${ }^{2,13}$. We chose to focus our studies on the bioremediation potential of $P$. anserina. This fungus only reproduces by sexual means, it is a non-pathogenic cosmopolitan species and its spread is easy to control. Targeted gene disruption experiments revealed that only one NAT, NAT2, is required for the growth and survival of the fungus in the presence of toxic AA. These findings provided a new basis for the bioremediation of AA-contaminated soils.

Given the detoxifying activity of NATs, the presence of NAT-encoding genes in many other fungi ${ }^{15}$ and the fungal biomass in soils, our studies show that fungal bioremediation of AA represents a promising perspective. Further studies are needed for setting up bioremediation protocols in natural conditions and to assess the possible effects of other soil fungi on AA biodegradation.

\section{Philippe Silar is at the Institut de Génétique et Microbiologie, CNRS UMR 8621 , Univ. Paris-Sud 11, 91405 Orsay cedex, and Univ. Paris Diderot, Sorbonne Paris Cité, UFR SdV, F-75205 Paris, France.}

Julien Dairou, Angélique Cocaign, Florent Busi, Fernando Rodrigues-Lima and Jean-Marie Dupret are at the Unité de Biologie Fonctionnelle et Adaptative (BFA), Univ. Paris Diderot, Sorbonne Paris Cité, BFA, EAC 4413 CNRS, F-75205 Paris, France.

Correspondence to P.S. and J.-M.D. e-mails:philippe.silar@igmors.u-psud.fr; jean-marie.dupret@univ-paris-diderot.fr
1. Harms, H., Schlosser, D. \& Wick, L. Y. Untapped potential: exploiting fungi in bioremediation of hazardous chemicals. Nature Rev. Microbiol. 9 177-192 (2011).

2. Martins, M. et al. An acetyltransferase conferring tolerance to toxic aromatic amine chemicals: molecular and functional studies. J. Biol. Chem. 284 , 18726-18733 (2009).

3. Kim, D. \& Guengerich, F. P. Cytochrome P450 activation of arylamines and heterocyclic amines. Annu. Rev. Pharmacol. Toxicol. 45, 27-49 (2005).

4. US National Toxicology Program. 11 th Report on Carcinogens. (US Department of Health and Human Services, 2005).

5. Ito, Y., Matsuda, Y. \& Suzuki, T. Effects of 3,4-dichloroaniline on expression of ahr2 and cyp 1 a 1 in zebrafish adults and embryos. Comp. Biochem. Physiol. C Toxicol. Pharmacol. 152, 189-194 (2010)

6. Lee, J. B. et al. Microbial biodegradation and toxicity of vinclozolin and its toxic metabolite 3,5-dichloroaniline. J. Microbiol. Biotechnol. 18, 343-349 (2008).

7. Garcia-Galan, M. J., Diaz-Cruz, M. S. \& Barcelo, D. Determination of 19 sulfonamides in environmental water samples by automated on-line solid-phase extraction-liquid chromatography-tandem mass spectrometry (SPE-LC-MS/MS). Talanta 81, 355-366 (2010).

8. Jin, C., Chen, Q., Sun, R., Zhou, Q. \& Liu, J. Eco-toxic effects of sulfadiazine sodium, sulfamonomethoxine sodium and enrofloxacin on wheat, Chinese cabbage and tomato. Ecotoxicology 18, 878-885 (2009).

9. Sartorius, M. et al. Sulphadimethoxine inhibits Phaseolus vulgaris root growth and development of $\mathrm{N}$-fixing nodules. Chemosphere 76, 306-312 (2009).

10. Khalid, A., Arshad, M. \& Crowley, D. E. Accelerated decolorization of structurally different azo dyes by newly isolated bacterial strains. Appl. Microbiol. Biotechnol. 78, 361-369 (2008)

11. Frische, T. Screening for soil toxicity and mutagenicity using luminescent bacteria - a case study of the explosive 2,4,6-trinitrotoluene (TNT). Ecotoxicol. Environ. Saf. 51, 133-144 (2002).

12. Tixier, C. et al. Biotransformation of phenylurea herbicides by a soil bacterial strain, Arthrobacter sp. N2 structure, ecotoxicity and fate of diuron metabolite with soil fungi. Chemosphere 46, 519-526 (2002).

13. Rodrigues-Lima, F. et al. Cloning, functional expression and characterization of Mesorhizobium loti arylamine $\mathrm{N}$-acetyltransferases: rhizobial symbiosis supplies leguminous plants with the xenobiotic $N$-acetylation pathway. Mol. Microbiol. 60, 505-512 (2006).

14. Dupret, J. M. \& Rodrigues-Lima, F. Structure and regulation of the drug-metabolizing enzymes arylamine $N$-acetyltransferases. Curr. Med. Chem. 12, 311-318 (2005).

15. Martins, M., Dairou, J., Rodrigues-Lima, F., Dupret, J. M. $\S$ Silar, P. Insights into the phylogeny or arylamine $\mathrm{N}$-acetyltransferases in fungi. J. Mol. Evol. 71, 141-152 (2010).

Competing interests statement

The authors declare no competing financial interests. 